\title{
Intrinsic hardness of crystalline solids
}

The current status of various theoretical approaches to the prediction of material hardness has been reviewed. It is shown that the simple empirical correlation with the shear moduli generally provide very good estimates of the Vickers hardness. Semi-empirical models based solely on the strength of the chemical bonds, although performed as well, are theoretically incomplete. First-principles calculations of the ideal stress and shear strength is perhaps the most reliable and theoretically sound approach available to compare theoretical predictions with experiment.

Key words: hardness, crystalline solids, shear moduli, strength of the chemical bonds, ideal stress, shear strength.

\section{INTRODUCTION}

"Hardness, like the storminess of the seas, is easily appreciated but not readily measured", commented H. O'Niell in the classic treatise on the measurement of the hardness of metals in 1934 [1]. In colloquial terms, the hardness of a material is the intrinsic resistance to deformation when a force is applied. Since force can be applied in various means, hardness is not a property that can be quantified easily in an absolute scale. The definition of hardness is complicated further by the distinction between microscopic and macroscopic strength. The microscopic hardness is dependent on the strength of atomic bonding. In contrast, the macroscopic strength of a real material is dominated by the behavior of dislocations or cracks. There are three common operational definitions [2]. Scratch and indentation hardness refer, respectively, to the resistance to plastic deformation due to a friction or a constant load from a sharp object. Rebound hardness is measured as the height of the bounce of an object dropped on the material and is related to the elasticity. The recent development of nanoindentation, where the mechanical behavior of a material is probed in a nanosized region of a surface beneath a nanoindenter, is expected to be less affected by cracks or dislocations, is most amendable for comparison with theoretical predictions employing modern electronic structure theory. However, special care is needed to be taken in the study of materials close to the hardness of diamond (superhard materials with hardness $>$ $60 \mathrm{GPa}$ ), since the indentation is no longer controlled by plastic deformation [3]. The possibility of brittle cracking and deformation of the indentation tip may affect the measured results. The "measured" hardness is dependent on to the type of an indenter, the applied loads, the time of the indentation, sample orientation and the quality of the tested surface. In view of these difficulties, there is no consensus how to characterize "ultrahardness" by a single number [3]. Similarly, a theoretical definition for hardness of a bulk crystal is also not easy to establish. Recently, several empirical models have been proposed to relate computable quantities to the Vickers hardness scale [4]. As in the experiment, this choice is not unique. The yield strength [5], which is the maximum stress that a crystal can attain under uniform deformation without extrinsic effects, is indicated by the maxima of a 
stress-strain curve. In principle, it can be simulated by electronic structure calculations and, perhaps, is the most appropriate working definition.

Regardless of the definition of hardness, in the absence of extrinsic impurities, the resistance of a material to deformation is determined by the strength of the local (nearest-neighbor) interatomic (bonding) interactions. In a covalent solid, the chemical bonds are localized and it is not unreasonable to expect that the compressibility (resistance to volume change), which is characterized by the bulk moduli, may be connected to the hardness [6]. In fact, diamond having the largest bulk modulus of all covalent solids is also the hardest bulk material in existence. This assumption, in the best scenario, is only valid when the forces are applied isotropically. This is not the case in indentation experiments where both normal (to the direction of force) and shear (parallel to the direction of force) stress are to be considered. In a sense the hardness of a crystal is the ability to resist plastic deformation from hydrostatic compression, tensile load and shear. From the empirical observation of the relationship of glide mobility and stress strength [7], it was suggested that the hardness of a material may be better correlated with the shear modulus. A comparison of the Vickers hardness with the bulk and shear moduli ( $G$, modulus of rigidity, the ratio of shear stress to shear strain) from a common set of covalent ceramic materials (oxides, nitrides, and metal carbides) are shown in Figs. 1, $a$ and $b$, respectively [8]. It is obvious that for the chosen set of data, the Vickers hardness shows a quasi-linear dependence on the shear modulus [8]. This is an important empirical relationship as it connects a computable quantity, the shear modulus, with the experimentally determined Vickers hardness. A similar linear correlation was also reported for metals [9]. The difference is that the slope of the line is two orders of magnitude less than that of the covalent solids. A fact that may be attributed to the weak delocalized metallic bonding [10]. For practical applications, it is desirable to establish a universal function that connects quantities predicted from First-Principles calculations with the measured hardness. This universal function should comprise all the essential factors governing the hardness and applicable to a wide range of materials with different bonding properties.

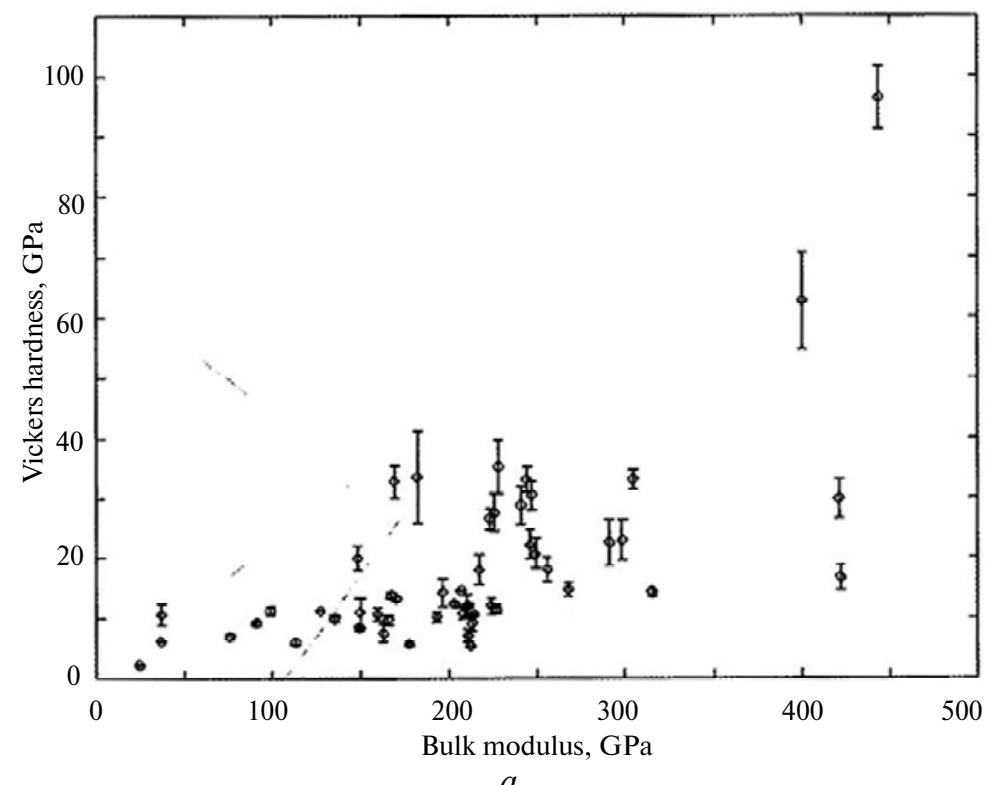

Fig. 1. Correlation of Vickers hardness with (a) bulk modulus and $(b)$ shear modulus. The dash line is a linear fit to the data (taken from [8]). 


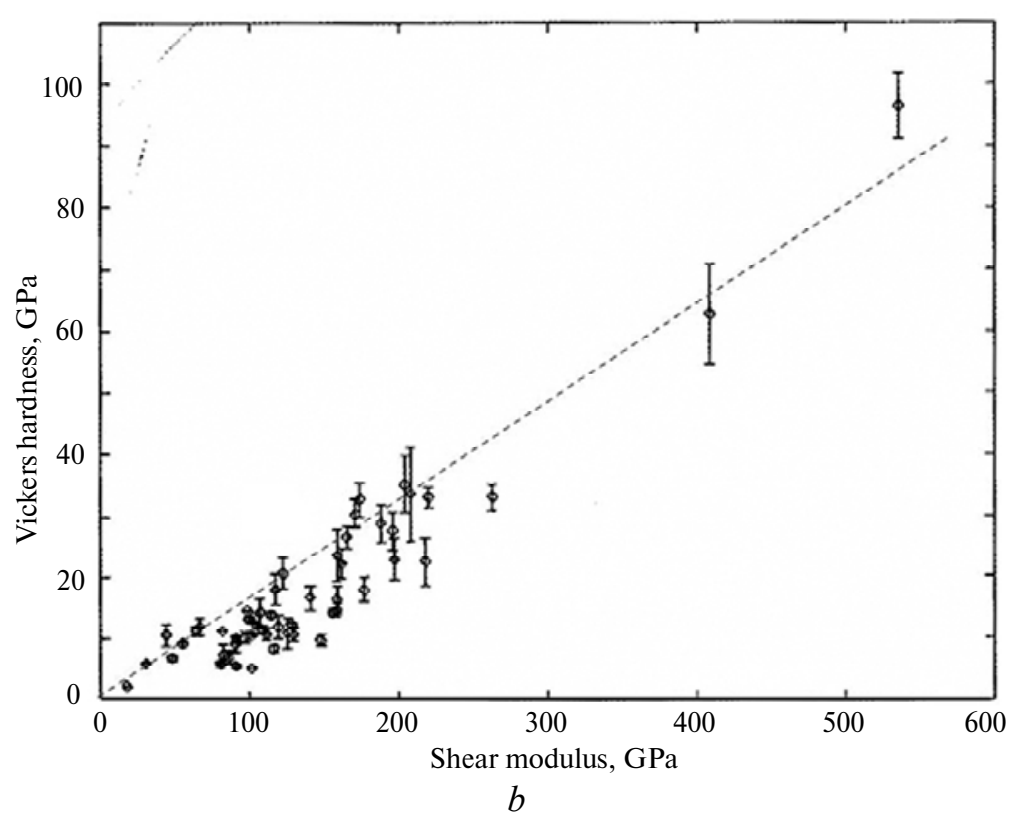

Fig. 1. (Contd.)

The outline of this paper is as follows. First, the basic principles of chemical bonding and the relationship to hardness will be presented. Phenomenological schemes connecting the properties of covalency-dominant solids to the hardness scale are presented. This is followed by a discussion on the extension of these schemes to nanostructured materials. First-principles calculations of ideal tensile and shear strengths of crystals will be reviewed. The paper is concluded with a brief summary.

\section{CHEMICAL PRINCIPLES OF HARDNESS}

The strength of a two center - two electron (Lewis) chemical bond between is determined by the exchange energy and is dependent on the overlap of the (hybridized) atomic orbitals of the respective atoms $[10,11]$. Consider a simple example of the interaction of two identical atoms (A) each contributing an atomic orbital to the bonding; in a tight binding description [11] and neglecting repulsion, the energy of the molecular orbitals is given by $E_{+}=\frac{E_{\mathrm{A}}^{0}+\Delta_{\mathrm{AB}}}{1+S_{\mathrm{AB}}}$ and $E_{-}=\frac{E_{\mathrm{A}}^{0}-\Delta_{\mathrm{AB}}}{1-S_{\mathrm{AB}}}$, where $E_{+}$and $E_{-}$are the energies of the bonding and antibonding orbitals, respectively. $S_{\mathrm{AB}}$ is the overlap integral, $E_{\mathrm{A}}^{0}$ is the effective potential of an electron in the atomic orbital of $\mathrm{A}$, and $\Delta_{\mathrm{AB}}$ is the energy associated with the two overlapping atomic orbitals. Taking the Taylor expansion to the first order, $E_{+} \approx E_{\mathrm{A}}^{0}+\left(\Delta_{\mathrm{AB}}-E_{\mathrm{A}}^{0} S_{\mathrm{AB}}\right)$ and $E_{-} \approx E_{\mathrm{A}}^{0}-\left(\Delta_{\mathrm{AB}}-E_{\mathrm{A}}^{0} S_{\mathrm{AB}}\right)$. Furthermore, it can be shown that $\Delta_{\mathrm{AB}} \propto-S_{\mathrm{AB}}<0$. Thus, the energy gap $\left(\Delta E=E_{-}-E_{+}\right) \approx$ $2\left(\Delta_{\mathrm{AB}}-E_{\mathrm{A}}^{0} S_{\mathrm{AB}}\right) \propto S_{\mathrm{AB}}$. Therefore, the better the overlap between the atomic orbitals, the larger the stabilization (bond) energy and the larger the energy gap. For a two electron bond, only the bonding orbital is occupied and the energy of the highest occupied molecular orbital (HOMO) is $E_{+}$, and that of the lowest 
unoccupied molecular orbital (LUMO) is $E_{-}$. Since both $E_{+}$and $\Delta E$ are dependent on the magnitude of the orbital overlap, it is a convenient indicator determining the strength of the chemical bond. In a covalent crystal, a collection of two center two electron chemical bonds is the stabilization factor for the crystal structure and determines the mechanical strength. Strong chemical bonds in a crystal not only resist isotropic compression but they also help to maintain the structural integrity from shear deformation.

The strength of a solid is determined by the yield stresses (e.g., indentation hardness), which are related to motions of the dislocations. The mobility of dislocation in a particular direction can be described as the gliding of two crystallographic planes against each other. In this process dislocation "kinks" are formed (Fig. 2) [9]. The stress needed to move a kink at a given velocity depends

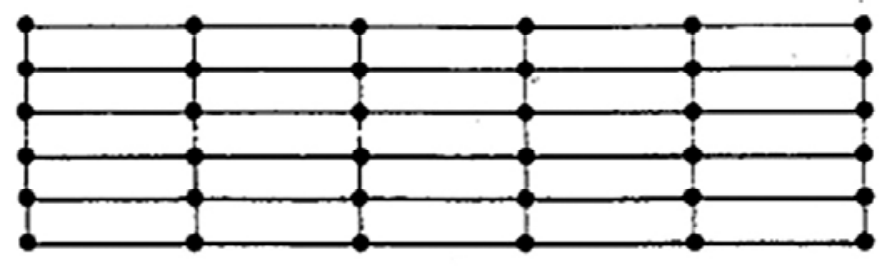

$a$


C

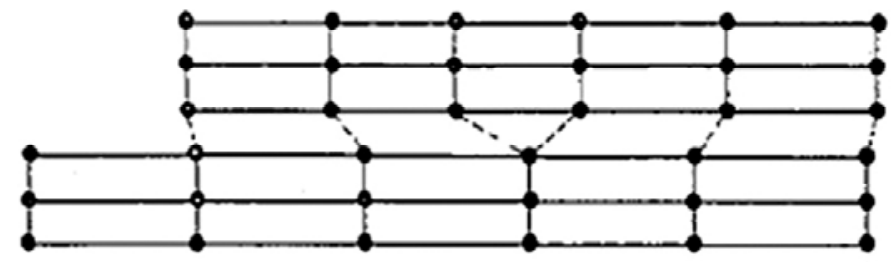

$d$



Fig. 2. Movement of a kink developed from the gliding of lattice plane under a shear strain, $(a)$ to (d) show the sequence of bond breakage and formation (adapted from [9]). 
on the energy of the kink and its position. Therefore, the energy involved in the movement of a dislocation kink is not unlike the energy path connecting the various stages of the gliding of two crystal planes shown in Fig. 2. In the same manner, the potential energy profile can be depicted intuitively or semi-empirically from the consideration of the breaking and formation of chemical bonds for a molecule. The diagram correlating the modification of molecular orbitals in this process is known as the Walsh diagram [12]. An illustration of the energy profile for a dislocation motion in a covalent solid is depicted in Fig. 3 [9]. Initially, the chemical bond is intact and the HOMO-LUMO energy separation is the largest. As the "kinks" migrate, the covalent bonds between the crystal planes weaken. As a result, the gap energy is reduced. Since the atoms on the two crystal planes have moved away from the optimum bonding positions, overlaps between atoms in the two planes are reduced and the stabilization energy $\left(E_{\mathrm{HOMO}}\right)$ is expected to diminish. Therefore, in shear deformation, $E_{\mathrm{HOMO}}$ should increase with a concomitant decrease in $E_{\text {LUMO }}$. As the product of the stress and strain is the work done on the system in the shear deformation, this quantity is related to the change in the energy of the HOMO $\left(E_{\text {Номо }}\right)$. Hence, the stress strength of a crystal is governed by orbital overlaps in the initial structure and at the displaced dislocation kink. If good overlap is maintained throughout the shearing, the solid will simply undergo plastic deformation. If the breaking of chemical bonds occurs for low stress deformation, the material will have low stress strength.

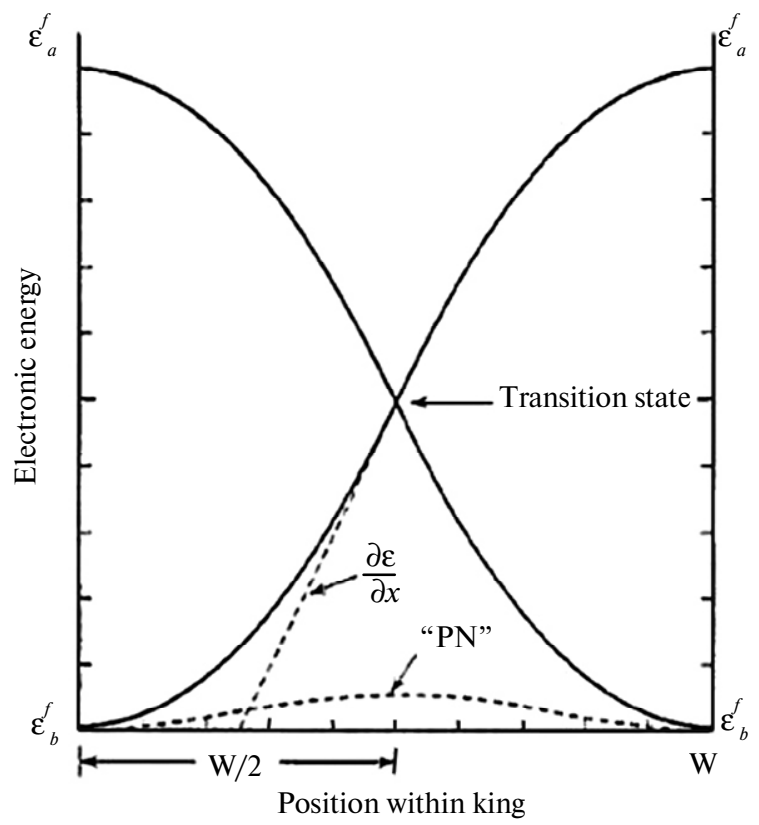

Fig. 3. Schematic orbital correlation diagram (Walsh diagram) depicting the change in HOMO $\left(\varepsilon_{a}\right)$ and LUMO $\left(\varepsilon_{b}\right)$ energy as a function of the displacement (W) of a kink (taken from [9]).

A convincing demonstration of the chemical principles presented above is the hardness enhancement observed in transition-metal carbonitrides [13]. From experiments it is found that the mixed alloy of cubic $\operatorname{TiC}_{x} \mathrm{~N}_{1-x}$ with a rock-salt structure achieves the maximum hardness when the valence electron concentration is about 8.4 per unit cell. Figure 4 shows the correlation of the measured microhardness in Vickers scale of $\mathrm{TiC}_{x} \mathrm{~N}_{1-x}$ with calculated shear modulus $\left(c_{44}\right)$ as a function of the valence electron concentration (VEC). The VEC was modified by 
varying the relative amount of $\mathrm{C}$ and $\mathrm{N}$ atoms. Theoretical shear moduli were calculated using the virtual crystal method where an effective pseudopotential was used to approximate disordered $\mathrm{C}$ and $\mathrm{N}$ by taking the weighted average of the atomic $\mathrm{C}$ and $\mathrm{N}$ pseudopotentials according to the stoichiometry. It is obvious that the maximum in $c_{44}$ corresponds to the highest Vickers hardness and occurs at a VEC of 8.4. Figure 5 shows the band structure of the reference TiC (i.e. $\operatorname{TiC}_{x} \mathrm{~N}_{1-x} x=1$ ) at

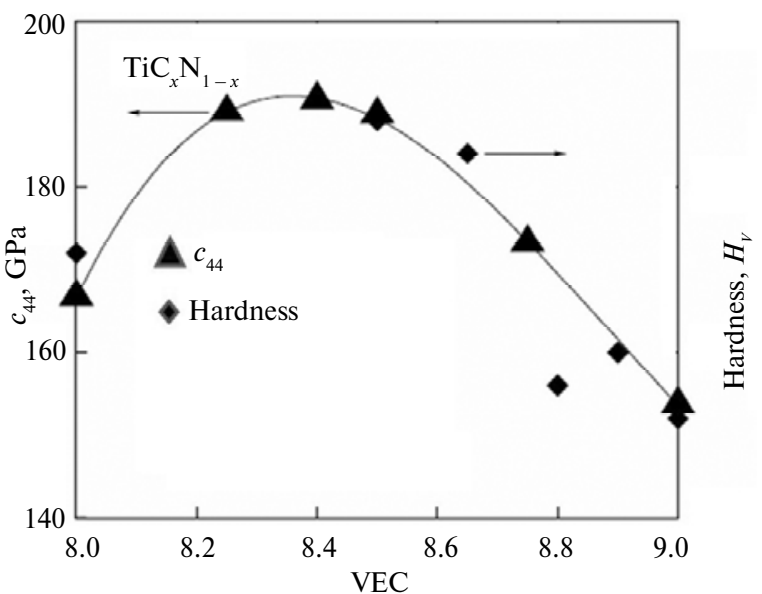

Fig. 4. Relationship of the valence electron count (VEC) with calculated shear modulus $\left(c_{44}\right)$ and experimental hardness $\left(H_{V}\right)$ for $\mathrm{TiC}_{x} \mathrm{~N}_{x}$ alloy of different compositions (taken from [13]). zero strain (Fig. 5, a) and under a shear strain $\varepsilon_{x y}=0.01$. The difference in the band structure is dramatic. Atfinite shear strain, the fourth electronic band increases in energy (thick dark line $\mathrm{K} \rightarrow \Gamma \rightarrow \mathrm{L}$ ), while the energy of the fifth band drops and crosses the Fermi level along $\mathrm{X} \rightarrow \Gamma$. Inspection of the valence charge density map (Fig. 6,a) shows that the fourth band is composed of mainly Ti $d_{x^{2}-y^{2}}-\mathrm{C}\left(p_{x}, p_{y}\right)$ bonding. A shear deformation in the $x y$ plane disrupts the bonding from the optimal configuration (Fig. 6,b) resulting in the rise of the fourth electronic band. On the other hand, Ti $d-d$ interactions in the fifth electronic band is enhanced by the shear strain due to the shortening of nearest neighbor $\mathrm{Ti}-\mathrm{Ti}$ separation in the [110] direction. The

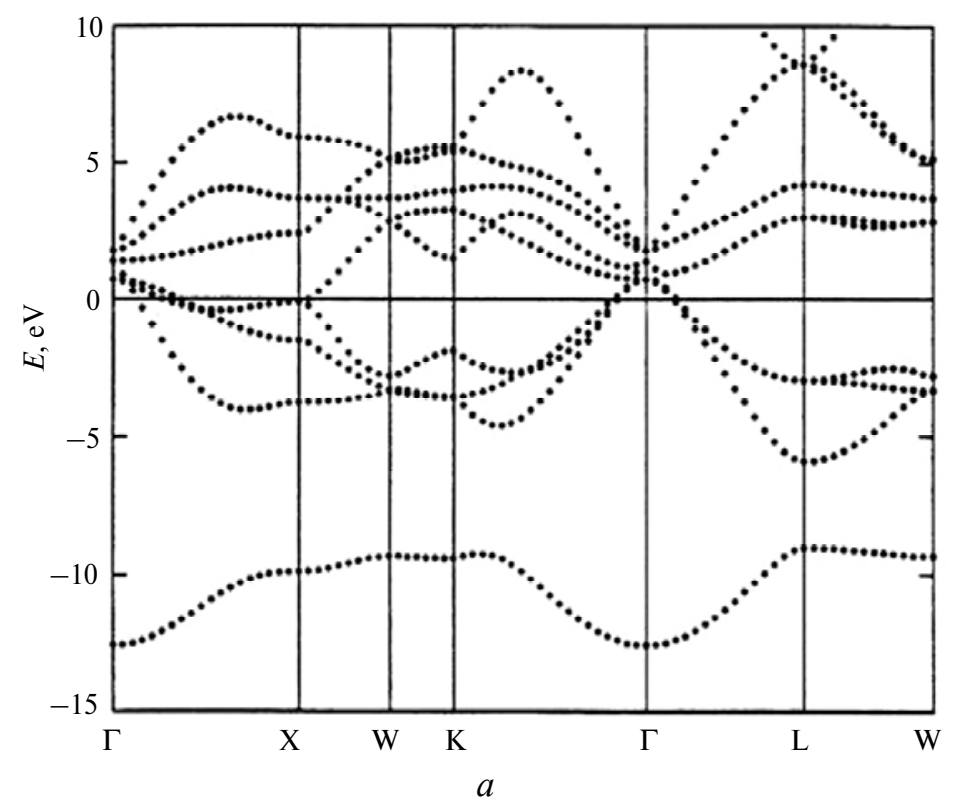

Fig. 5. Band structure of $\operatorname{TiC}(a)$ equilibrium and $(b)$ at strain $\varepsilon_{x y}=0.01$. Note the changes in the dispersion of the 4th (thick line) and 5th electronic band (taken from [13]). 


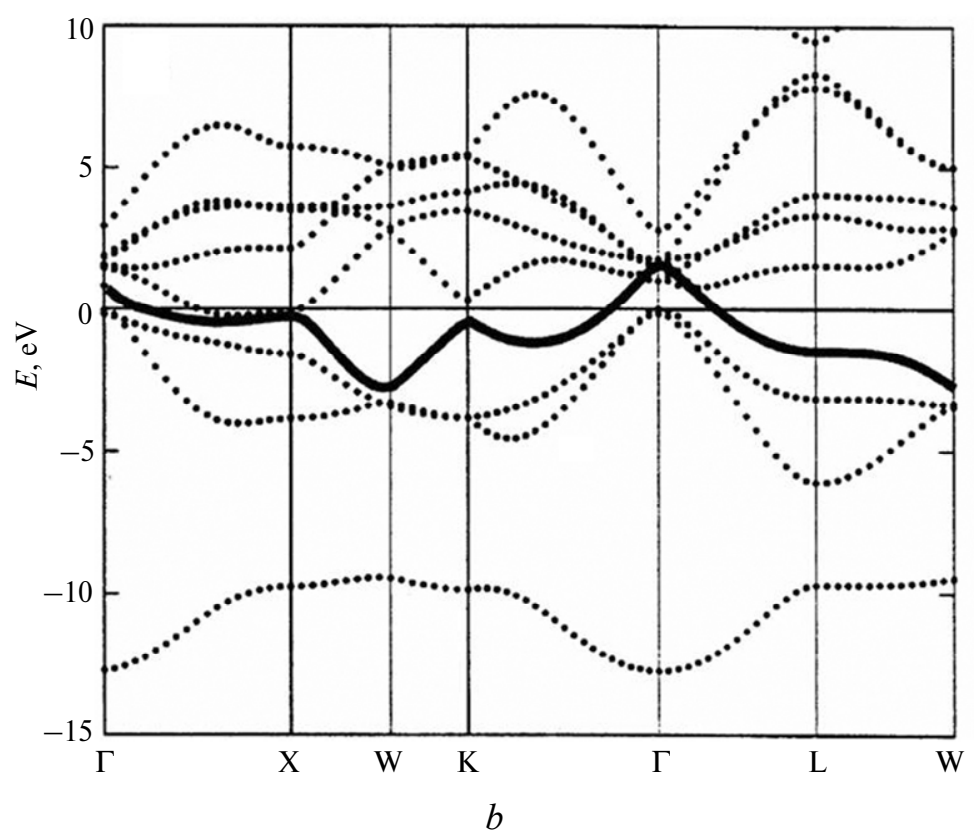

Fig. 5. (Contd.)

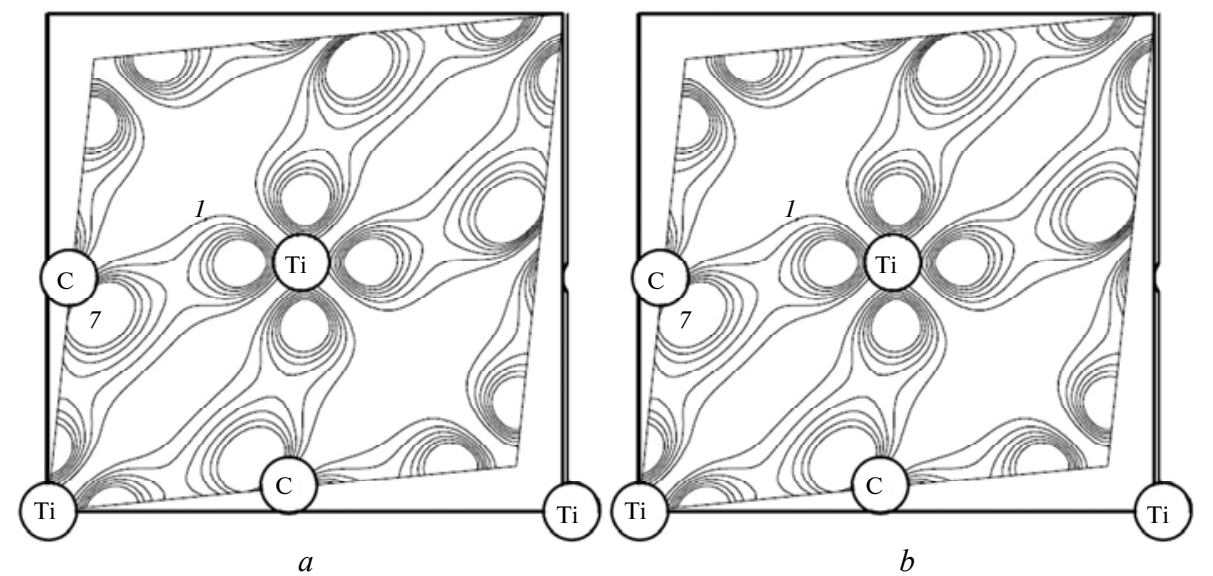

Fig. 6. Charge density of the 4 th and 5 th electronic band of TiC under strain $\left(\varepsilon_{x y}=0.01\right)$ (taken from [13]).

replacement of $\mathrm{C}$ by $\mathrm{N}$ increases the number of valence electrons. In a rigid band description, the additional electrons occupy the empty states of the fourth band near $\Gamma$, raising the Fermi level and strengthening the $\mathrm{Ti}-\mathrm{C}$ interactions, which results in a higher $c_{44}$. The complete filling of the shear-resistive bonding states is at a VEC $\sim 8.4$, which corresponds to the observed maximum in the experimental microhardness. This is strong evidence indicating that this is the cause for the enhancement of hardness in $\mathrm{TiC}$ via the replacement of $\mathrm{C}$ by $\mathrm{N}$.

\section{PHENOMENOLOGICAL SCHEMES}

\section{Bulk modulus}

The first empirical scheme for the estimation of the bulk moduli of covalent solids was developed by Cohen [14]. The scheme is based on the Phillips-Van 
Vechten energy scheme for the characterization of the covalent and ionic nature of tetrahedral bonded solids $[15,16]$. It was argued that the bulk modulus of semiconductors with the diamond or zinc-blende structure is related to a homopolar energy gap $E_{h}$. Using a scaling relationship between $E_{h}$ and the nearest-neighbour distance $d$, an empirical formula [17] that reproduced a number of tetrahedral bonded semiconductors was found, $B=\frac{(19.71-2.20 \lambda)}{d^{3.5}}$, with $B$ in Mbar and $d$ in

$\AA$. The parameter $\lambda$ accounts for the ionicity and for homopolar semiconductors $\lambda=0$; for heteropolar Group III-V and II-VI $\lambda=1$ and 2 , respectively. As has been noted above, bulk modulus is not an appropriate parameter for the estimation of hardness due to the neglect of dislocations. Nevertheless, the Phillips-van Vachten energy scheme is being employed in recent formulations relating the hardness to the strength of a chemical bond. A notable success is the method suggested by Gao et al. [18], which will be discussed in the next section.

\section{Insulators}

Hardness is not a computable quantity. However, for practical purposes it would be very convenient to relate the measured hardness of a material to some of the fundamental properties. Gao et al. proposed a scheme linking the Vickers hardness for a broad class of covalent insulating solids to their macroscopic properties, which can be obtained from the first-principles calculations. The basic premise of this approach is the extension of the chemical principles where the plastic deformation of a covalent solid creates motion of dislocations. The barrier, or resistance force, to the plastic glide is related to the number of covalent bond $\left(N_{a}\right)$, the solid and the corresponding energy gap $E_{g}$. This simple assumption is reasonable as it relates the intrinsic hardness $\left(H_{a}\right)$ to the bond strength $\left(E_{g}\right)$ and the coordination number [18],

$$
H(\mathrm{GPa})=A N_{a} E_{g},
$$

where $A$ is the proportional constant, which can be determined from fitting the expression to a standard set of materials with known Vickers hardness. $N_{a}$ can be evaluated from the electron number density, $N_{e}$, as

$$
N_{a}=\left(\sum_{i} n_{i} Z_{i} / 2 V\right)^{2 / 3}=\left(N_{e} / 2\right)^{2 / 3},
$$

where $n_{\mathrm{i}}$ is the number of the $i$ th atom in the cell and $Z_{i}$ is the number of a valence electron of the $i$ th atom. Following Phillip, $E_{g}$ for binary polar covalent $\mathrm{AB}_{m}$ crystal is separated into covalent homopolar gap $E_{h}$ and an ionic or heterpolar gap $C[15,16]$,

$$
E_{g}^{2}=E_{h}^{2}+C^{2} .
$$

The homopolar energy gap can be estimated from the empirical expression $E_{n}=39.75 d^{-2.5}$, where $d$ is the interatomic distance. The ionic contribution to the energy gap is deduced from $E_{h}$ by treating ionicity as a simple screening effect. This is accounted for by introducing a correction factor $\exp \left(-\alpha f_{i}\right)$ to describe the screening effect for each bond, where $f_{i}=1-E_{h}^{2} / E_{g}^{2}$ is the ionicity of the chemical bond in crystal in the Phillips scale. To determine the constant $A$ in Eq. (1), $H_{V} / E_{h} N_{a}$ is plotted against $f_{i}$ using a test of matereials with known 
hardness. An equation relating the hardness in the Vickers scale to $f_{i}, N_{e}$ and $d$ is then obtained.

$$
H_{V}(\mathrm{GPa})=556 \frac{N_{a} e^{-1.191 f_{i}}}{d^{2.5}}=350 \frac{N_{e}^{2 / 3} e^{-1.191 f_{i}}}{d^{2.5}} .
$$

It is straightforward to extend the formula to multicomponent systems. The average hardness is assumed to be the geometrical mean of the different types of covalent bonds in the system. Therefore,

$$
H_{V}=\left[\prod^{\mu}\left(H_{V}^{\mu}\right)^{n^{\mu}}\right]^{1 / \sum n^{\mu}}
$$

where $H_{V}^{\mu}$ is the hardness of the $\mu$-type bonds, $n^{\mu}$ is the number of bonds of type 0 . A comparison of the calculated and observed Vickers hardness for selected covalent solids is shown in Fig. 7. In view of the significant spread of measured hardness (over $100 \mathrm{GPa}$ ), the overall agreement is highly satisfactory. In recent years, Eq. (4) and its variant Eq. (5) have been used extensively for the prediction of hardness for a large class of proposed superhard materials from the firstprinciples calculation.

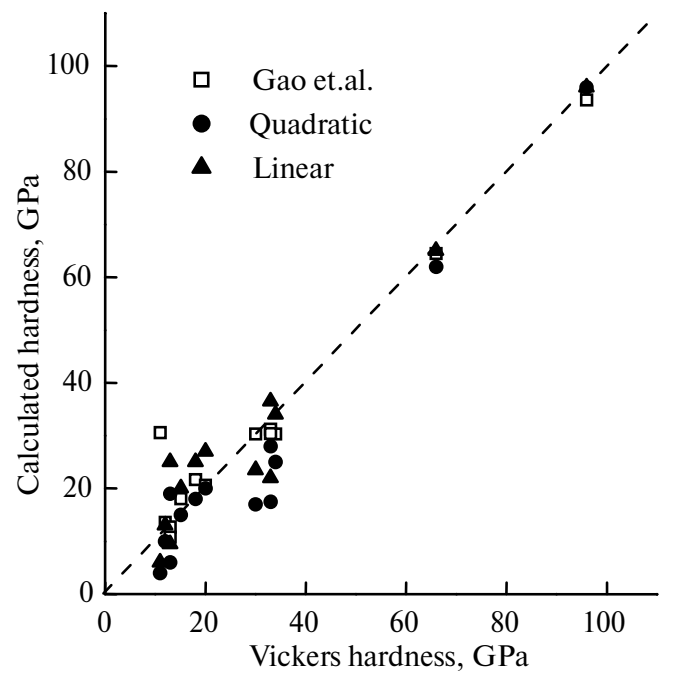

Fig. 7. Correlation of calculated and measured Vickers hardness values (see the text).

Does the success of Eq. (4) owe to the incorporation of all essential physics contributing to the hardness or simply due to the success of fitting to a large training set on the determination of the empirical parameters? A deeper appreciation of the derivation of the formula reveals that the implicit assumptions do not differ from the basic concept for the bulk modulus (vide supra), where the hardness is attributed solely to the magnitude of the energy gap. This is obviously not sufficient, since the hardness is also determined by the shear strength. A complete theory must take into account the full elasticity of the material. Unfortunately, no theoretical or empirical relationships correlating the energy gap of a covalent solid with its elastic moduli, particularly, the shear modulus $(G)$ [19] has ever been established. There are several definitions for shear modulus. 
Throughout this paper, except indicated, the convention of Voigt is adopted. To investigate this point further, in Fig. 7, the hardness of single component and binary covalent crystals used in the determination of the empirical coefficients in Eq. (4) were estimated from the correlation shown in [8] (Fig. 1,b) employing the experimental shear moduli. To establish the correlation quantitatively, the data in Fig. 1b are approximated by linear and quadratic functions. From the fitted functional forms, the Vickers hardness can be estimated from the knowledge of the shear modulus. The results are summarized in Fig. 7 together with the corresponding values obtained using Eq. (4). The mutual agreement between the two different empirical schemes and the overall agreement with experimental hardness are striking. This observation indicates that although (shear) elasticity was not explicitly included, after fitting to experimental data, the model of Gao [18] apparently includes this effect implicitly. On the other hand, the strong correlation shows that hardness can also be estimated from the shear modulus. First-principles calculations of elastic constants are now routine [20] and conversions to shear moduli for all crystal classes are well known [19]. The two methods (i.e. Eq. (4) and hardness-shear modulus correlation) are complementary, both can be used to estimate the hardness for new materials. However, for a given crystal, when using Eq. (4), the structure of the crystal and the energy gaps $E_{h}$ and $E_{g}$ must be known. On the other hand, the correlation shown in Fig. 1, $b$ can be used with calculated or measured shear modulus.

To explore the reliability of the empirical correlation for ternary compounds, the hardness of $\beta-\mathrm{BC}_{2} \mathrm{~N}$ [18] is estimated from Fig. 1, $b$. No experimental shear modulus is available but the calculated $G$ of $\beta-\mathrm{BC}_{2} \mathrm{~N}$ is $300-320 \mathrm{GPa}$. The corresponding Vickers hardness estimated from Fig. $1, b$ is $42-51 \mathrm{GPa}$. In comparison, the hardness calculated by Eq. (5) is $78 \mathrm{GPa}$. It should be noted that the experimental hardness of $\beta-\mathrm{BC}_{2} \mathrm{~N}$ is not known yet. In [8], the calculated hardness was compared to an experimental value for $\mathrm{cBC}_{2} \mathrm{~N}$ of $76 \mathrm{GPa}$ [21]. Using an observed shear modulus of $\mathrm{cBC}_{2} \mathrm{~N}$ of $450 \mathrm{GPa}$, the hardness was estimated to be 54-74 GPa from Fig. 1, $b$. The good agreement with experiment suggests that the correlation of hardness with shear modulus is reliable.

An alternate scheme for predicting hardness without the use of empirical parameters has been proposed by Simunek and Vackar [22]. This method is an extension of the hardness relationship of Eqs. (4) and (5). Instead of using empirical rules to determine ionicity and the energy gaps, a bond strength index $S_{i j}$ between atoms $i$ and $j$ is defined,

$$
S_{i j}=\sqrt{e_{i} e_{j}}\left(d_{i j} n_{i j}\right),
$$

where $e_{i}=Z_{i} / R$ is a reference energy, $Z_{i}$ is the valence electron number of atom $i$, and $n_{i j}$ is the number of bonds between atom $i$ and its neighboring atoms $j$ at the nearest neighbor distance $d_{j j}$. Radius $R_{i}$ for each atom in a crystal is determined such that the radius $R_{i}$ of the sphere contains exactly the charge $Z_{i}$. Instead of relating the hardness to bond energy gaps, it is assumed that hardness is proportional to the bond strength $S_{i j}$. In this way, no empirical parameters are needed and the hardness can be determined solely from the first principles calculations. Good agreement between the theory and experiment was found [22].

\section{Nanostructures}

An advantage of having an expression relating the hardness to the fundamental properties is that it can be extended to other systems. Recent experiments have 
shown that nanocrystalline solids, such as BN [23] or diamond [24], possess hardness "higher" than bulk diamond. These observations contradicted the reversed Hall-Petch effect that the hardness and yield stress should decrease with the grain size. This argument is supported by a computer simulation, which suggested that in nanocrystallites, the plastic deformation is mainly due to a large number of small sliding at the grain boundaries rather than the motion of dislocations. The softening in the shear stress is a result of the larger fraction of atoms at the grain boundaries [25]. In contrast, novel phenomenon of hardening of nanocrystallites can be explained by combining the empirical theory of hardness with the effect of quantum confinement [26]. In nanocrystals, the conduction/valence band edges shift generally to higher energy relative to the bulk material when the crystalline size is decreased. According to the Kubo theory [27], the bandgap $E_{g, \text { nano }}$ of a nanocrystal should increase inversely with the volume $V$

$$
E_{g, \text { nano }}=E_{g, b u l k}+\delta \text {, }
$$

where $E_{g, b u l k}$ is an "effective" band gap of the bulk material and $\delta$ is the energy shift due to quantum confinement given by [27]

$$
\delta=\frac{2 \hbar^{2} \pi^{2}}{m V\left(3 \pi^{2} N_{e}\right)^{1 / 3}},
$$

where $m$ is the atomic mass and $N_{e}$ is the electron density. Equation (4) can be extended to include quantum confinement effects, the energy shift for a nanocrystallite $\delta p$ can be written as,

$$
\delta_{p}=K \frac{2 \hbar^{2} \pi^{2}}{m V\left(3 \pi^{2} N_{e}\right)^{1 / 3}}=\left(K \frac{2 \hbar^{2} \pi^{2}}{m V\left(3 \pi^{2}\right)^{1 / 3}}\right) N_{e}^{-/ 3}=f(D) N_{e}^{-1 / 3},
$$

where parameter $K$ may also be a function of the particle diameter. We can approximate the term in the brackets of Eq. (9) as a function of cluster diameter $D$, $f(D)$. Equating the band shift ratio to experimental shift ratio, $\delta_{p} / E_{g p}=\delta / E_{g}$ and using CdS as a calibrant [28], the bracketed term in Eq. (9) can be evaluated. It is found that an empirical formula, $\delta_{p}=\frac{24.0}{D N_{e}^{1 / 3}}$ with $\delta_{p}$ (in $\mathrm{eV}$ ) inversely proportional to cluster diameter $D$, gave a good fit to the experimental data of nanocrystalline CdS. Finally, an expression for the hardness of a nanocrystal is obtained by substituting Eq. (9) into Eq. (1) [26]

$$
H(\mathrm{GPa})=A N_{a}\left(E_{g, \text { bulk }}+\delta_{p}\right)=A N_{a}\left(E_{g, b u l k}+24.0 / D N_{e}^{1 / 3}\right) .
$$

This equation has been used to compute the hardness of nano-3C- and 6diamonds and the results are summarized in table. The trend and the predicted hardness are consistent with experimental observation.

Recently, a unique superhard aggregated boron nitride nanocomposites (ABNNCs) showing the enhancement of hardness up to $100 \%$ in comparison with a cBN single crystal has been synthesized and characterized [29]. The decrease of the grain size down to nano- and subnanolevel results in enormous mechanical property enhancement with maximum hardness of $85 \mathrm{GPa}$. This finding contradicts the reverse Hall-Petch effect [25]. However, a fit of the measured hardness $\left(H_{V}\right)$ to the crystallite size $(d)$, according to the Hall-Petch equation $[29,30]\left(H_{V}=H_{0}+\right.$ $K / \sqrt{d}$ ), which has an inverse square root relationship with $d$ was found to be 
unsatisfactory. However, adding the quantum effects term (Eq. (9)), which have a $1 / d$ dependence, to the Hall-Petch equation improves the agreement with the observed trend significantly over a large hardness range on the crystallite size (14-2000 nm) (Fig. 8) [29].

\section{Calculated Vickers hardness for $6 \mathrm{H}$ and $3 \mathrm{C}$ diamond nanocrystallites}

\begin{tabular}{cc}
\hline Phase & $H_{V \text { cal nano }}(\mathrm{GPa})$ \\
\hline 6H diamond & $96(5 \mathrm{~nm})$ \\
& $94(12 \mathrm{~nm})$ \\
3C diamond & $108(1.245 \mathrm{~nm})$ \\
& $97(5 \mathrm{~nm})$ \\
& $95(12 \mathrm{~nm})$ \\
& $112(1.0 \mathrm{~nm})$ \\
\hline
\end{tabular}

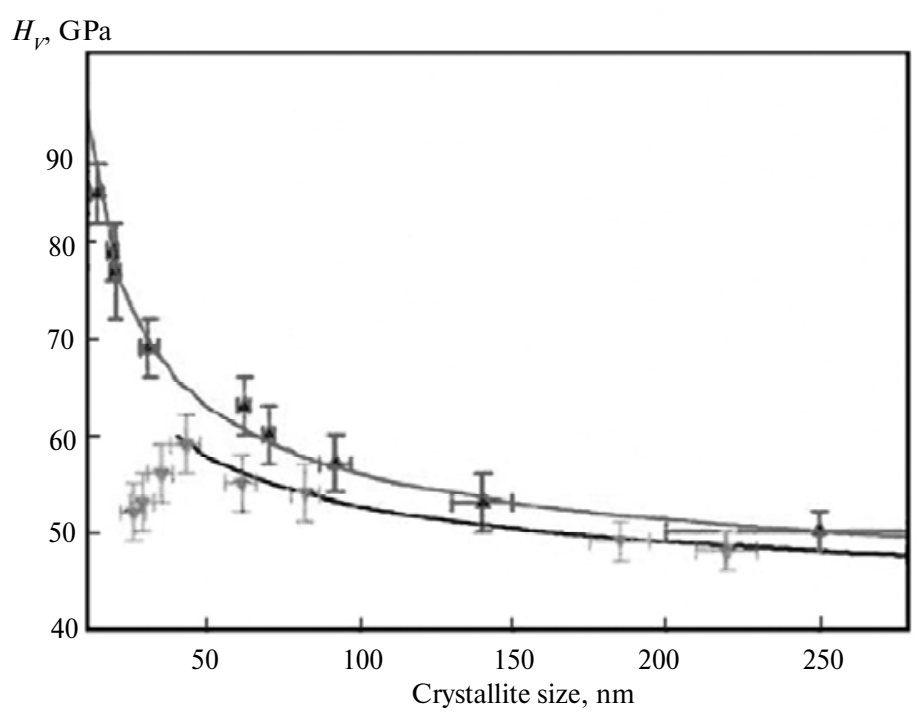

Fig. 8. Comparison of calculated and experimental Vickers hardness $\left(H_{V}\right)$ using the Hall-Petch equation including the correction for quantum confinement (modified from [29]).

\section{Metals}

The theoretical basis of "metallic" bonding is still under intense debate [31, 32]. In one school of thought [32] a metal may be described as comprised of weak covalent (directions) bonds and delocalized electrons. The weak covalent bonds help to define the crystal structure. This point of view is clearly demonstrated in the elucidation of the structure and electronic properties of $\mathrm{Li}-\mathrm{Al}$ alloys [33]. This description of metal structure becomes even more relevant to hard, weakly metallic compounds. An extension of Eq. (4) to transition metal carbides and nitrides has been proposed [34]. The metallicity $\left(f_{m}\right)$ is related to the number of thermally excited electrons $\left(n_{m}\right)$ above the Fermi level at temperature $T$ and represent as a product of the electron density of states at the Fermi level, $D_{f}$ and $k_{B} T$, where $k_{B}$ is the Boltzmann constant. At room temperature, $f_{m}=0.026 D_{f} / n_{e}$, where $n_{e}$ is the total number of valence electrons. It was postulated [34] that the existence of both ionic and metallic contributions will reduce the covalent bond density $N_{a}$ (Eq. (1)). An empirical correction factor to the ionicity $f_{i}$ is then added, 


$$
f_{i}=\left[1-\exp \left(-\left|P_{C}-P\right| / P\right]^{0.635},\right.
$$

where $P$ is the overlap population of a bond and $P_{c}$ is the overlap population of a bond in a hypothetical pure covalent crystal with the same structure. In addition, an empirical correction to the screening effect $\left(e^{-\beta f_{m}^{n}}\right)$ is included. The final expression is similar to Eq. (4) where the Vickers hardness is

$$
H_{V}(\mathrm{GPa})=A^{\prime} N_{a} e^{-1.191 f-\beta_{m i}^{n}} E_{h} .
$$

Here, the constants $A^{\prime}, \beta$ and $n$ were determined from fitting to known hardness from a selected set of compounds [34].

The expression (Eq. (12)) is not entirely satisfactory for a number of reasons. Conceptually, it is not possible to define an energy gap $\left(E_{h}\right)$ for a "bond" in metals. Overlap population [11] is not a well defined quantity. It is well-known that it is dependent on the system and then criteria on dividing the total overlap among two atoms [11]. Finally, the constants appearing in Eq. (12) were determined from overlap population computed with CASTEP code and are not easily transferable to



Fig. 9. Correlation of calculated and measured Vickers hardness (see the text) for covalent dominant transition metal carbides and nitrides. be used by other electronic codes. The universality and usefulness of this expression remains to be tested.

A comparison of the hardness estimated from the shear moduli and using Eq. (12) is shown in Fig. 9 along with experimental data. For metallic carbides and nitrides the range of hardness is small. It is no surprise that Eq. (12), which was fitted with the experimental data, gave the best agreement. Note that the shear moduli used in the preparation of Fig. 9 include both experimental and theoretical values and the two sets of data may be subjected to errors $(> \pm 10 \%)$. It is observed that the experimental trend is correctly reproduced by both the linear and quadratic fits to Fig. 1, $b$, except that the hardness values obtained from the linear fit are somewhat higher. In passing, it is noteworthy that the Vickers hardness for the recently synthesized "ultraincompressible" $\mathrm{OsB}_{2}$ [35] from the hardness-shear modulus curve using the theoretical $G$ of $206 \mathrm{GPa}$ is $25-31 \mathrm{GPa}$ [36]. This value is comparable to $35 \mathrm{GPa}$ [37] calculated with the parameter - free expression Eq. (6) and the experimental estimate of $37 \mathrm{GPa}$ [38]. The good agreement between the hardness values obtained from the parameter - free Eq. (6) with experiment may suggest that it is preferable over the semi-empirical scheme. The simple hardness-shear modulus correlation (Fig. 1,b) can also be used as a qualitative guide.

\section{Ab intio calculations of ideal tensile and shear strength}

The ideal strength is the stress where a dislocation-free crystal becomes unstable and undergoes spontaneous plastic deformation. This sets the upper bound on the mechanical strength of a material. Detailed analysis [39] of the mechanics of 
nanoindentation has shown that after proper consideration of the crystallography of loading and the correction of the nonlinearity of the elastic response at large strains, the measured values can be compared quantitatively to the results of the first-principles calculations. In the calculations, the ideal strength is defined as the maximum stress in the stress-strain curve in the weakest tensile stretch or shear slip direction [40, 41]. Since first-principles ideal strength calculations explore the stress-strain energy profile at large structural deformation, changes in the electronic structure under strain are also correctly reproduced. Therefore, unlike the use of bulk and shear moduli obtained at equilibrium to estimate the hardness, ideal strength calculations provide accurate tensile and shear strengths and, from the analysis of the results, the mechanism leading to structural failure can be revealed.

To compute the ideal strength of a crystal, one needs to determine the weakest tensile direction. This is accomplished by calculation of the tensile stresses along candidate crystallographic directions $[40,41]$. For a given tensile direction, the relevant lattice vectors are deformed in the direction of the applied strains. At each step, all the atom positions are relaxed and the cell basis vectors are adjusted to remove stresses orthogonal to the applied strains. Once the weakest tensile direction is found, the critical shear stress is calculated by applying shear deformation in the "easy"-slip plane perpendicular to this direction. A typical theoretical calculated stress-strain curve with tensile applied at different crystallography directions is shown in Fig. 10 [42].

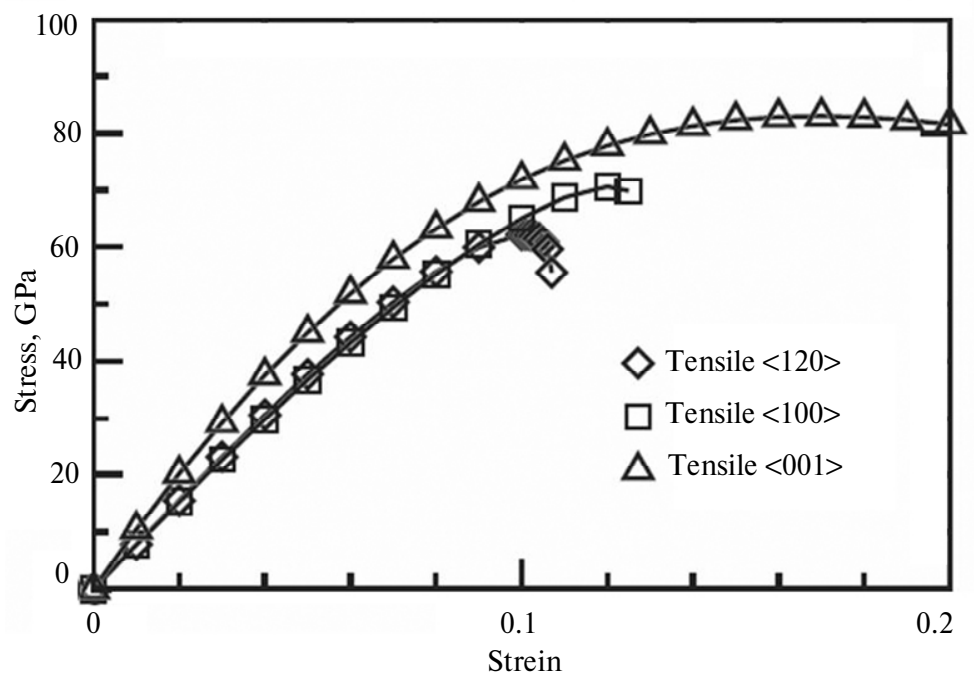

Fig. 10. Theoretical stress-strain relationship for $\beta-\mathrm{BC}_{2} \mathrm{~N}$ (taken from [42]).

The ideal strengths of many metals, metal alloys, hard ceramic materials and nanostructures have been studied with $a b$ inito calculations [41]. The method has also been applied to potential superhard materials such as transition metal carbides and nitrides, carbon nitrides, and boron carbides. A good example is the recently synthesized "ultra"-incompressible $\mathrm{OsB}_{2}$ [35]. It was shown from high pressure Xray diffraction that the bulk modulus has an unusually high value of $365 \mathrm{GPa}$ [35]. This value is to be compared with diamond (442 GPa) and Os metal (395$462 \mathrm{GPa}$ ). Moreover, the as-synthesized powder has been shown to scratch the polished surface of a sapphire crystal indicating that the hardness of this material should exceed $20 \mathrm{GPa}$ [35]. This surprising finding has stimulated many theoretical 
studies. From a calculated shear modulus of $205 \mathrm{GPa}$ using gradient corrected density functional, the Vickers hardness was estimated to be $31.8 \mathrm{GPa}$ [37]. The measured hardness from nanoindentation under different loadings varies between 30 and $37 \mathrm{GPa}[38,43]$. All indications point to $\mathrm{OsB}_{2}$ may be a superhard material.

Calculations of the ideal tensile and shear strengths of $\mathrm{OsB}_{2}$ have been reported in [44]. $\mathrm{OsB}_{2}$ is formed from alternate layers of Os and $\mathrm{B}$ atoms. Examination of the crystal structure suggests that the bonding in the (001) Os1-Os2 plane is the weakest and the strength against shear deformation will be the lowest in the [010] direction (Fig. 11). The calculated tensile stresses in several symmetric crystallographic directions are shown in Fig. 11. Not surprisingly, the highest tensile strength is predicted along [100] and the lowest peak of the stress is at [011]. A calculated ideal tensile strength in the [011] direction of $25.9 \mathrm{GPa}$ is still higher than that of iron (12.6 GPa) [45]. The covalent Os-B bonds enhance the resistance of $\mathrm{OsB}_{2}$ to tensile deformation in all stress directions.
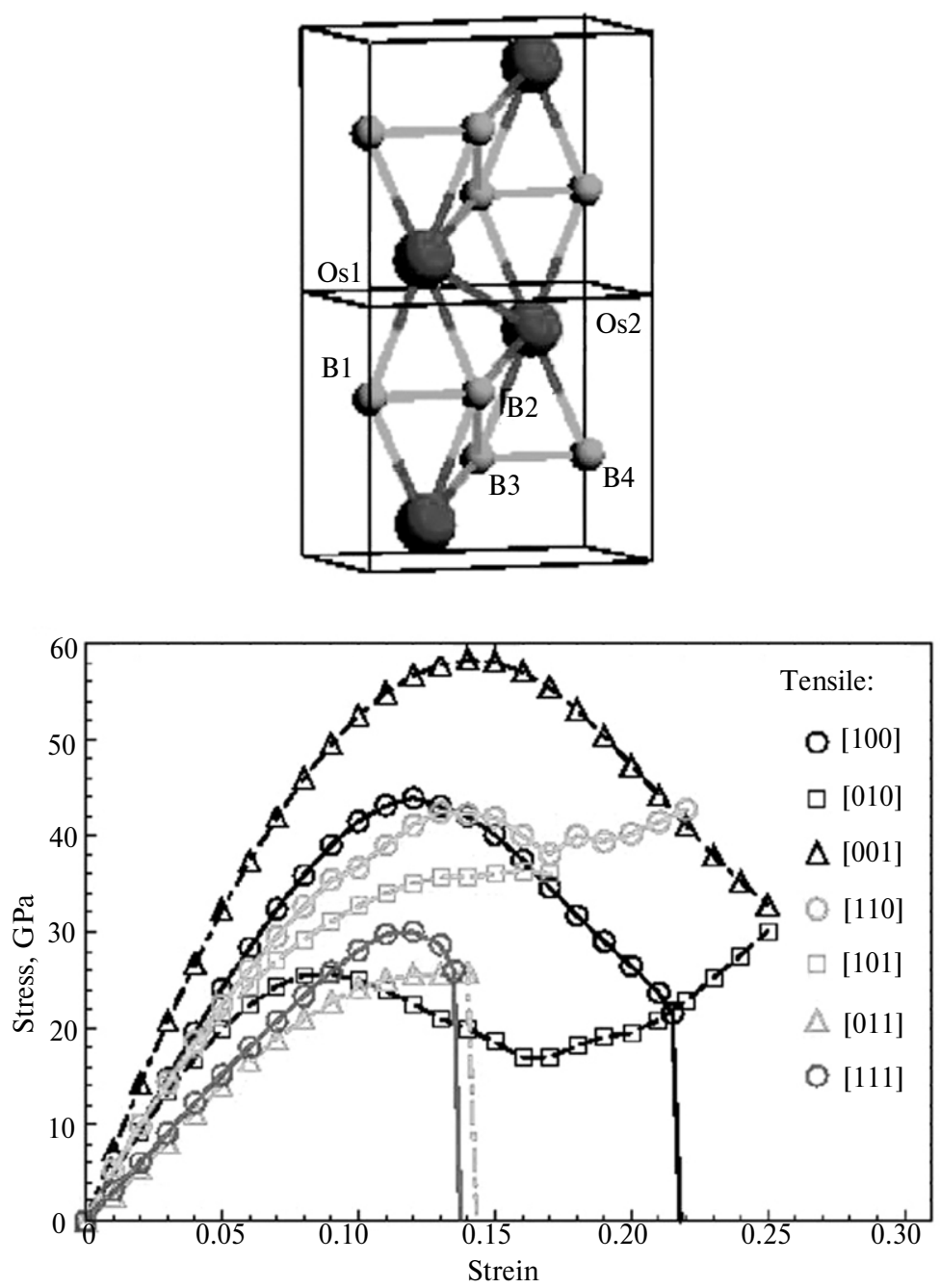

Fig. 11. Crystal structure $(a)$ and calculated tensile stress-strain curves for $\mathrm{OsB}_{2}$ (adapted from [44]) (b). 
Figure 12 shows calculated shear stresses in the (001) plane along the three principal crystallographic axes. The shear strength is highly anisotropic. Maxima in the stress-strain curves are found at 26.9, 19.4, and 9.1 GPa in the [001], [010], and [100] directions, respectively. Therefore, the lowest ideal shear stress is in the (001)[010] shear direction. The highly anisotropic strength in the (001) plane is related to the crystal structure where the Os- $\mathrm{B}$ bonds only help to strengthen the resistance against shear deformations in the (001)[100] direction. The inspection of the stress-strain curves also provides information on mechanical properties of $\mathrm{OsB}_{2}$. The discontinuities in the stress along (001)[100] and (001)[110] shear directions are indicative of a brittle material, where the chemical bonds broke abruptly. This contrasts with the continuous stress-strain relationship along the (001)[010] shear direction, which is typical for a ductile material. The ductility can be understood from the analysis of the evolution of the crystal structure during shear deformation. Four instantaneous snapshots taken along the (001)[010] shear are shown in the lower panel of Fig. 12. The Os-B bonds were maintained throughout the deformation and the crystal planes glided against each other and did not offer strong resistance against the shear strain. At first glance this conclusion is perhaps counter-intuitive. However, this observation can be rationalized as follows. Strong Os - B covalent interactions only provide strong repulsive force to resist the bond compression and enhancing the tensile strengths. However, when sheared in the (001)[100] direction, the Os-B distance is extended somewhat but the overlaps are still maintained. The calculated ideal strength in the (001)[010] shear direction is only slightly higher than that of iron $(7.2 \mathrm{GPa})$. Incidentally, a maximum shear strength along the (001)[100] of $26.9 \mathrm{GPa}$ is comparable to a measured Vickers hardness of $30 \mathrm{GPa}$ on the (001) plane [43]. The example
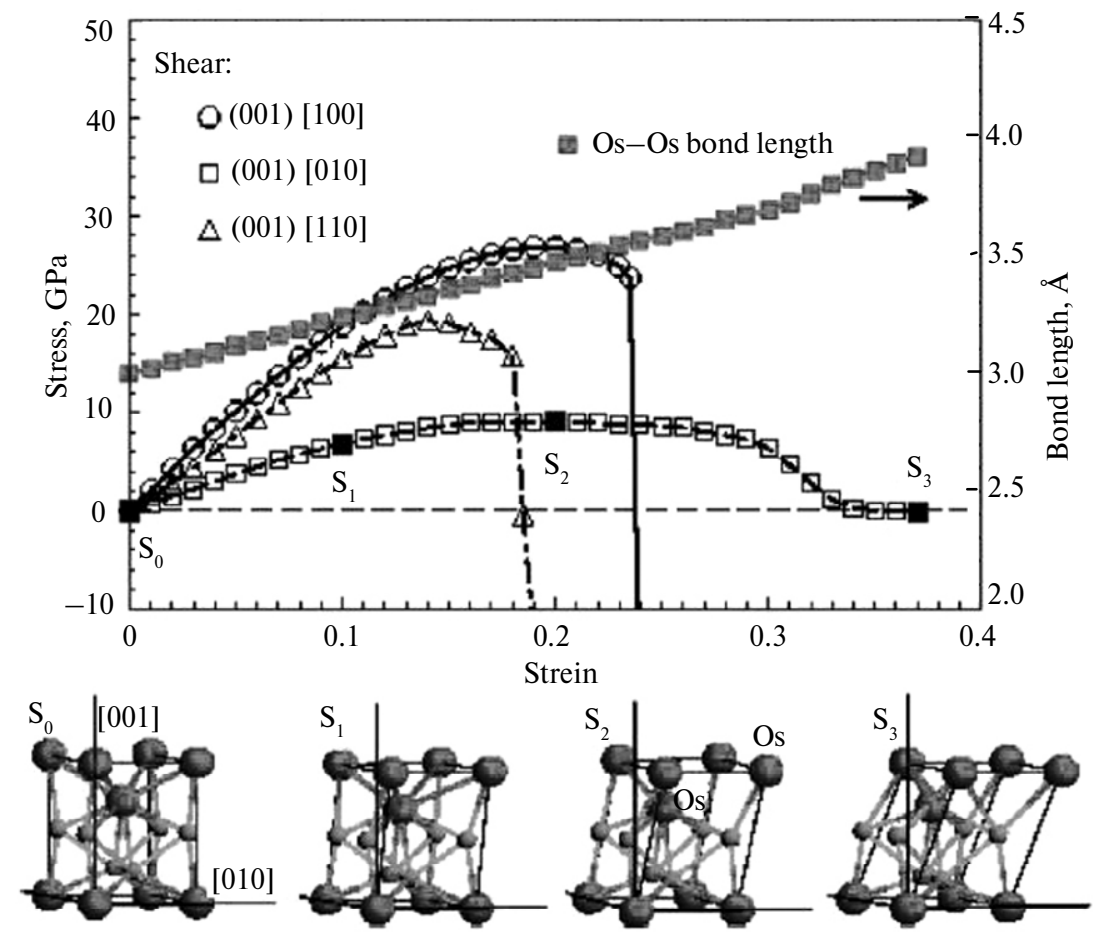

Fig. 12. Calculated stress-strain relationships for $\mathrm{OsB}_{2}$ at shear deformation in the [100], [010] and [110] crystal planes. The bottom figure shows the structures at the deformation points indicated in the corresponding (001)[010] stress-strain curve (taken from [44]). 
presented above illustrates that the first-principles ideal strength calculations can yield numerical results, which can be compared directly with nanoindentation experiments and provide detail information to rationalize the mechanical processes involved in shear deformations. It also highlights the importance and advantage on the characterization of hardness from the examination of crystal and the electronic structure accompanying the deformation processes. This information is, of course, not available from simple correlation with the shear moduli at the equilibrium structure or from parameter - free phenomenological or semi-empirical schemes.

\section{CONCLUSIONS}

In closing, to illustrate the usefulness and insight provided by the state-of-theart computational approaches, it is useful to remark the theoretical studies on a recently discovered superhard cubic boron carbide $\left(\mathrm{cBC}_{5}\right)$ [46]. A number of theoretical techniques have been applied to characterize the structure and hardness of this novel material. Since experimental results indicated that the B boron atoms are not aggregated (clustered), the first theoretical investigation focused on stable structures that may explain the diffraction pattern and the unusual hardness. Based on this assumption, algebraic evaluation of possible structures followed by calculations of the total energies, Raman spectra, and diffraction patterns led to the conclusion that B is randomly distributed in the cubic diamond structure [47]. An important result is that the ordered trigonal $(P 3 \mathrm{~m} 1)$ structure proposed earlier [48] is energetically not favorable and the calculated diffraction pattern is in conflict with experiment. A Vickers hardness of $79 \mathrm{GPa}$ for the B-disordered structure was estimated from the empirical shear moduli - hardness correlation, which is remarkably close to an observed value of $79 \mathrm{GPa}$ [47]. Subsequently, the disordered structure is also confirmed to be the most stable and best candidate for $\mathrm{cBC}_{5}$ from more elaborate special quasi-random structure (SQS) search procedure [49]. The ideal mechanical strength on model $\mathrm{BC}_{5}$ structures has also been investigated by two separate groups $[50,51]$. Both calculations demonstrate that the $P 3 m 1$ structure [48] has weak slip directions along $(10 \overline{1})$ and $(1 \overline{2} 1)$ in the [111] plane and cannot be a candidate for $\mathrm{cBC}_{5}[50,51]$. Moreover, the calculated ideal shear stresses are found to be much stronger (almost three times) on cubic structural models with randomly distributed $\mathrm{B}$ without clustering [51], again supporting a disordered structure.

A brief overview on the current status of the computational approaches for the determination and prediction of the material hardness was presented. The empirical scheme of correlating Vickers hardness with the shear modulus was found to perform reasonably well. The correlation is by no means quantitative. However, within the accuracy of measured hardness and shear moduli, the overall agreement is deemed to be highly satisfactory. There is no doubt that strong covalent bonding is a principal factor for ultra- and superhard materials. Phenomenological schemes assuming that the hardness is primarily governed by the strength of chemical bonds have been developed for covalent solids. These schemes that provide the conduits for direct comparison with experimental data are easy to use and proven to be reliable for a wide class of materials. Potential problems on extending these phenomenological schemes to metallic solids are highlighted. The most rigorous theoretical approach for studying hardness is through the calculations of ideal tensile and shear strength with first-principles electronic methods. This approach allows quantitative comparison with the results obtained from nanoindentation experiments and provides physical insights into the deformation mechanisms. The principal ingredient for a hard material is strong covalent chemical bonds. 
However, as demonstrated for the case of $\mathrm{TiC}_{x} \mathrm{~N}_{1-x}$ alloy [13], orbital rehybridization can also play a crucial role in determining the resistance to shear deformation. Ideal shear strength calculations have also shown that $[42,45]$ weaknesses in crystallographic planes determine the lattice instability and, therefore, the intrinsic hardness. To this end, the real space orbital correlation approach (e.g., Fig. 3) $[9,12]$ is a very useful conceptual and qualitative guide to predict hardness of a potential material. The search for high hardness and strength materials is important for a wide variety of industrial applications such as cutting tools, wear-resistant coatings and abrasive applications. Understanding the fundamental principles governing the hardness of a material is a prerequisite for the search and rational design of new materials. This is particularly important in view of recent advances in the development of efficient electronic codes and methods for structural prediction from first-principles.

Розглянуто сучасний стан різних теоретичних підходів до прогнозування твердості матеріалів. Показано, що проста емпірична кореляція з модулем зсуву звичайно дає дуже хорошу оцінку твердості за Віккерсом. Розроблені також напівемпіричні моделі, основані виключно на мічності хімічного зв'язку, є теоретично неповними. Розрахунки з перших принципів ідеального напруження $і$ міцності на зсув $\epsilon$, мабуть, найбільш надійним $і$ теоретично обтрунтованим підходом для порівняння теоретичних прогнозів з експериментальними результатами.

Ключові слова: твердість, кристал, модуль зсуву, міцність хімічного зв'язку, ідеальне напруження, міцність на зсув.

Рассмотрено современное состояние различных теоретических подходов $\kappa$ прогнозированию твердости материалов. Показано, что простая эмпирическая корреляция с модулем сдвига обычно дает очень хорошую оченку твердости по Виккерсу. Разработанные также полуэмпирические модели, основанные исключительно на прочности химических связей, являются теоретически неполными. Расчеты из первых принципов идеального напряжения и прочности на сдвиг представляют собой, вероятно, наиболее надежный и теоретически обоснованный подход для сравнения теоретических прогнозов с экспериментальными результатами.

Ключевые слова: твердость, кристалл, модуль сдвига, прочность химических связей, идеальное напряжение, прочность на сдвиг.

1. O'Neill H. The hardness of metals and its measurement - London: Chapman and Hall, 1934.

2. Malzbender J. Comment on hardness definitions // J. Euro. Ceramics Soc. — 2003. — 23. P. 1355.

3. Brazhkin V., Dubrovinskaia N., Nicol M. et al. What does "harder than diamond" mean? // Nature Materials. - 2004. - 3, N 9. - P. 576-577.

4. Fischer-Cripps $C$. Introduction to contact mechanics. - Heidelberg: Springer, 2000.

5. Beer F. P., Johnston J. E., Russell D., Dewolf J. T. Mechanics of materials / 3rd ed. McGraw-Hill, 2001.

6. Cohen M. L. Predicting new solids and their properties // Philos. Trans. R. Soc., London, Ser. A. - 1991. - 334, N 1635. - P. 501-513.

7. Nabarro R. N. Theory of crystal dislocation / Dover ed. - New York: Dover Publications, 1987.

8. Teter D. M. Computational alchemy: the search for new superhard materials // Mater. Res. Soc. Bulletin. - 1998. - 23. - P. 22-27.

9. Gilman J. J. Physical chemistry of intrinsic hardness // Mater. Sci. Eng. A. - 1996. — 209. - P. 74-81.

10. Pauing L. The nature of chemical bond. - Ithaca, New York: Cornell University Press, 1939.

11. Albright T. A., Burdett J. K., Whangbo M.-Y. Orbital interactions in chemistry. - New York: John Wiley \& Sons, 1984.

12. Walsh A. D. The electronic orbitals, shapes, and spectra of polyatomic molecules. Part III. HAB and HAAH molecules // J. Chem. Soc. (London). — 1953. — P. 2288 - 2296. 
13. Jhi S-H., Ihm J., Louie S. G., Cohen M. L. Electronic mechanism of hardness enhancement in transition-metal carbonitrides // Nature. — 1999. — 399. — P. 132.

14. Cohen M. L. Calculation of bulk moduli of diamond and zinc-blende solids // Phys. Rev. B. - 1986. - 32, N 12. - P. 7988-7991.

15. Phillips J. C. Bonds and bands in semiconductors. - New York: Academic Press, 1973.

16. Phillips J. C. Ionicity of the chemical bond in crystals // Rev. Mod. Phys. - 1970. - 42, N 3. - P. 317-356.

17. Liu A. and Cohen M.L. Prediction of new low compressibility solids // Science. - 1989. 245. - P. 841-842.

18. Gao F. M., He J. L., Wu E. D. et al. Hardness of covalent crystals // Phys. Rev. Lett. -2003. - 91. - P. 015502.

19. Grimvall G. Thermophysical properties of materials. - Amsterdam: Elsevier, 1999.

20. Martin R. M. Electronic structure: Basic theory and practical methods. - Cambridge: University Press, 2004.

21. Solozhenko V. L., Dub S. N., Novikov N. V. Mechanical properties of cubic $\mathrm{BC}_{2} \mathrm{~N}$, a new superhard phase // Diamond Relate Mater. — 2001. — 10, N 12. — P. 2228 - 2231.

22. Simunek $A$. and Vackar J. Hardness of covalent and ionic crystals: First-principle calculations // Phys. Rev. Lett. - 2006. - 96. - P. 085501.

23. Rafaja D., Klemm V., Motylenko M. et al. Synthesis, microstructure and hardness of bulk ultrahard BN nanocomposites // J. Mat. Res. — 2008. — 23. - P. 981 - 993.

24. Irifune T., Kurio A., Sakamoto S. et al. Ultrahard polycrystalline diamond from graphite // Nature. - 2003. - 421. - P. 599-600.

25. Schiotz J., Di Tolla F. D., Jacobsen K. W. Softening of nanocrystalline metals at very small grain sizes // Nature. - 1998. - 391. - P. 561 - 563.

26. Tse J. S., Klug D. D., Gao F. M. Hardness of nanocrystalline diamonds // Phys. Rev. B. 2006. - 73. - P. 140102

27. Harlperin W. P. Quantum size effects in metal particles // Rev. Mod. Phys. - 1986. - 58. - P. 533.

28. Wang Y., Herron N. Quantum size effects on the exciton energy of CdS clusters // Phys. Rev. B. - 1990. - 42. - P. 7253.

29. Dubrovinskaia N., Solozhenko V. L., Miyajima N. et al. Superhard nanocomposite of dense polymorphs of boron nitride: Noncarbon material has reached diamond hardness // Appl. Phys. Lett. - 2007. — 99, N 9. - P. 101921.

30. Hall E. $O$. The deformation and ageing of mild steel: III discussion of results // Proc. Phys. Soc. London, Sect B. - 1951. - 64. - P. 747.

31. Allen L. C., Capitani J. F. What is the metallic bond? // J. Amer. Chem. Soc. - 1994. 116. - P. 8810.

32. Burdett J. K., Czech P. T. What is the metallic bond? // J. Amer. Chem. Soc. - 1994. 116. - P. $8808-8809$.

33. Rousseau R., Tse J. S. Rationalization of structures of binary alloys in a real space atomic level perspective // Prog. Theoret. Phys. — 2000. — 138, Supplement. - P. 47.

34. Guo X., Li L., Liu Z. et al. Hardness of covalent compounds: Roles of metallic component and $d$ valence electrons // J. Appl. Phys. - 2008. - 104. - P. 023503.

35. Cumberland R. W., Weinberger M. B., Gilman J. J. et al. Osmium diboride, an ultraIncompressible, hard material // J. Amer. Chem. Soc. - 2005. - 127. - P. $7264-7265$.

36. Hao $X$., $X u$ Y., Wu Z. et al. Elastic anisotropy of $\mathrm{OsB}_{2}$ and $\mathrm{RuB}_{2}$ from first-principles study // J. Alloys Compounds. - 2008. - 453, N 1-2. - P. 413-417.

37. Chen X-Q., Fu C. L., Kremar M., Painter G. S. Electronic and structural origin of ultraincompressibility of $5 d$ transition-metal diborides $\mathrm{MB}_{2}(\mathrm{M}=\mathrm{W}, \mathrm{Re}, \mathrm{Os}) / /$ Phys. Rev. Lett., 2008. - 100. - P. 196403 (4 p.).

38. Chung H-Y., Yang J. M., Tolbert S. H., Kaner R. B. Anisotropic mechanical properties of ultra-incompressible, hard osmium diboride // J. Mater. Res. - 2008. - 23, N 6 . P. $1797-1801$

39. Krenn C. R., Roundy D., Cohen M. L. et al. Connecting atomistic and experimental estimates of ideal strength // Phys. Rev. B. - 2002. - 65. - P. 134111 (4 p.).

40. Roundy D., Kernn C. R., Cohen M. L., Morris J. W., Jr. The ideal strength of tungsten // Philos. Mag. A. - 2001. - 81. - P. 1725- 1747.

41. Ogata S., Umeno Y., Kohyama M. First-principles approaches to intrinsic strength and deformation of materials: perfect crystals, nano-structures, surfaces and interfaces // Modeling Simul. Mater. Sci. Eng. — 2009. - 17, N 1. - P. 013001. 
42. Zhang Y., Sun H., Chen $C$. Ideal tensile and shear strength of $\beta-\mathrm{C}_{3} \mathrm{~N}_{4}$ from first-principles calculations // Phys. Rev. B. - 2007. — 76, N 14. - P. 144101 (6 p.).

43. Chung H. Y., Weinberger M. B., Levine J. B. et al. Synthesis of ultra-incompressible superhard rhenium diboride at ambient pressure // Science. - 2007. - 316, N 5823. P. 436- 439 .

44. Yang J., Sun H., Chen C. Is osmium diboride an ultra-hard material? // J. Amer. Chem. Soc. - 2008. - 130, N 23. - P. 7200-7201.

45. Clatterbuck D. M., Chrzan D. C., Morris $J . W ., J r$. The ideal strength of iron in tension and shear // Acta Mater. - 2003. - 51. - P. 2271-2283.

46. Solozhenko V. L., Kurakevych O. O., Andrault D. et al. Ultimate metastable solubility of boron in diamond: Synthesis of superhard diamondlike $\mathrm{BC}_{5} / /$ Phys. Rev. Lett. — 2009. 102, N 1. - P. 015506 (4 p.).

47. Yao Y., Tse J. S., Klug D. D. Crystal and electronic structure of superhard $\mathrm{BC}_{5}$ : Firstprinciples structural optimizations // Phys. Rev. B. — 2009. - 80, N 9. - P. 094106 (6 p.).

48. Calandra M. and Mauri $F$. High- $T_{c}$ superconductivity in superhard diamondlike $\mathrm{BC}_{5} / /$ Phys. Rev. Lett. - 2008. - 101, N 1. - P. 016401 (4 p.).

49. Jiang C., Lin Z., Zhao $Y$. Superhard diamondlike $\mathrm{BC}_{5}$ : A first-principles investigation // Phys. Rev. B. - 2009. - 80, N 18. - P. 184101 (6 p.).

50. Lazar P., Podloucky R. Mechanical properties of superhard $\mathrm{BC}_{5} / /$ Appl. Phys. Lett. — 2009. — 94, N 25. - P. 251904 (3 p.).

51. Zhang R. F., Veprek S., Argon A. S. Effect of nanometer-sized grains on the superhardness of c-BC 5 : A first-principles study // Phys. Rev. B. — 2009. — 80, N 23. - P. 233401 (4 p.).

Department of Physics and Engineering Physics,

Received 25.11.09

University of Saskatchewan 